\title{
Cornell Medical Index Score Observed among Japanese Nursing Students
}

\author{
AKIKO FUKAHARA, NAOKO KODAMA, CHIE NAGAHIRO \\ SOMSONG NANAKORN $*, * *$ AND RYOKO OSAKA \\ School of Nursing, *Department of Public Health, Kurume University \\ School of Medicine, Kurume 830, Japan and ${ }^{*}$ Central Research Unit, \\ Faculty of Medicine, Khon Kaen University, Khon Kaen 40002, Thailand
}

Received for publication September 3, 1997

\begin{abstract}
Summary: Purpose of this study was to survey subjective health problems occurring among Japanese nursing students. Data collected with the self-administered Cornell Medical Index Health Questionnaire Japanese version (JCMI) from 102 Japanese first year nursing students were analyzed. The total CMI score (section A-R) ranged from 2-108 with an average of 23.1 $(\mathrm{SD}=15.9)$. The mean CMI for physical (section A-L) and psychological health complains (section $\mathrm{M}-\mathrm{R})$ were $15.7(\mathrm{SD}=11.8)$ and $7.3(\mathrm{SD}=6.1)$, respectively. Two-way analysis of variances with multiple comparison of means revealed significant relationships between age and psychological health complaints and both physical and overall health complaints. The older nursing students reported more physical and overall health complaints than the youngest. The nursing students who reported more complaints about psychological health also reported significantly more physical and overall health complaints. A comparison of Fukamachi's and Brodman's criteria for classification of neurotic traits is discussed. Findings from this study support the hypothesis that psychological health is somewhat reacted to physical health.
\end{abstract}

Key words Cornell Medical Index, physical health complaints, psychological health complaints, overall health complaints, nursing student, Japan

\section{Introduction}

The health status of nursing students has become a very important factor in health care services development. Hence, assessment of health problems of nursing students is useful not only for the individual but also for the level of nursing education and development of health care services.
Mental health problems are increasingly prevalent among nursing students studying in some parts of Japan (Sakamoto et al. 1986).

This study aims at screening subjective health problems occurring among Japanese nursing students by the self-administered Cornell Medical Index Health Questionnaire (CMI).

Correspondence to: Ryoko Osaka, School of Nursing, Kurume University, 777-1 HigashiKushihara machi, Kurume 830, Japan. 


\section{Materials and Methods}

The research instrument was the Cornell Medical Index Health Questionnaire Japanese version (J-CMI). The CMI is a self-administered health questionnaire which was originally developed in the United States as an aid to the clinician (Brodman et al. 1949). It has been translated into several languages and widely used as a sensitive indicator of a person's health and neurotic tendency in several countries including Japan (Brodman et al. 1952a; Abramson, 1966; Kanehisa and Fukamachi, 1988). It consists of 195 simply worded questions grouped into 18 sections (section A to R). Sections A-l, hereafter termed physical health complaints, include 144 questions pertinent to physical symptoms related to the subject's \& family's health history and habits. Sections M-R, hereafter termed psychological health complaints, include 51 questions pertinent to mood and feeling patterns. The detailed structure is shown in Fig. 1. Each question is answered 'yes' or 'no', scored as one point or zero, respectively. A Yes-response answer indicates an individual's currently or previously experienced symptoms (Brodman et al. 1949). The J-CMI is freely available and is widely used in clinical practice as a screening procedure to evaluate neurotic tendencies (Kanehisa and Fukamachi, 1988).

An individual's CMI score is determined by the sum of yes-responses over the whole questionnaire. Mean CMI for a population is the sum of the individual's CMI scores divided by the total number of subjects.

The subjects of the present study were

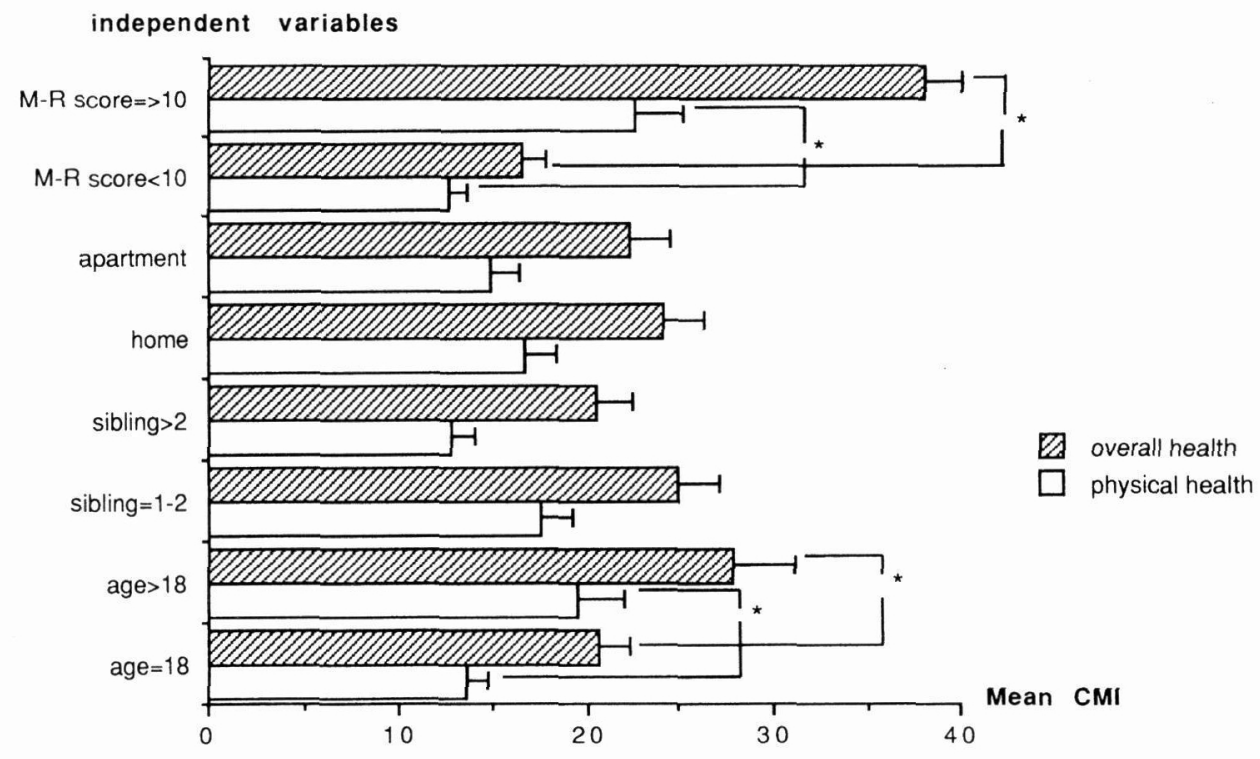

Fig. 1. Mean CMI by independent variables.

* indicates significant difference at the $5 \%$ level. 
Japanese nursing students in the first yearclass of the schools of nursing located in Kyushu, southern Japan. All the students were instructed to honestly self-administer the J-CMI. Of the 106 students in this class, 102 (96.2\%) completed the questionnaire. Data collection was performed in July 1995, which was the last month of the school year's first semester.

Reliability of the J-CMI was tested in terms of its internal consistency using Cronbach coefficient alpha (1951). Relationships between demographic variables and CMI scores were examined using two-way analysis of variance (ANOVA). Calculated $95 \%$ confidence intervals were used to detect statistical significance. Data analyses were conducted using the SAS statistical package at the Computer Center, Kurume University School of Medicine, Japan.

\section{Results}

\section{Demographic characteristics}

Mean age of the subjects was 18.4 $(\mathrm{SD}=0.5)$ years, with age range between 18-21 years. About one third (34.3\%) of the subjects were over 18 years of age. The number of siblings ranged from 1-4, with an average of $2.4(\mathrm{SD}=0.6)$, and $59.8 \%$ of the subjects had 1-2 siblings. Almost half of them (44.1\%) lived with their families, the others lived alone in rented apartments.

\section{Reliability of the J-CMI}

There was significant correlation between the total CMI score and its grouped sections' scores $\left(r_{p}=0.94\right.$ for section A-L, and 0.78 for section M-R), as well as between scores of section A-L and section M-R $\left(r_{p}=0.53\right)$. Cronbach coefficient alpha was 0.89 which indicates adequate internal consistency.

\section{CMI scores, mean CMI}

Individual CMI scores in this sample of 102 subjects ranged from 2 to 108 with an average of $23.1(\mathrm{SD}=15.9)$.

Mean CMI for physical health complaints and psychological health complaints were $15.7(\mathrm{SD}=11.8)$ and 7.3 $(\mathrm{SD}=6.1)$, respectively.

\section{Patterns of complaints}

Table 1 presents patterns of complaints indicated by percentage of yesresponse by section. The highest percentage of complaints among physical and

TABLE 1.

Percentage of yes-response by section of the total subjects

\begin{tabular}{lrc}
\hline \multicolumn{1}{c}{ Section referring to } & $\begin{array}{c}\text { Number of } \\
\text { questions }\end{array}$ & $\begin{array}{c}\text { Percentage } \\
(\mathrm{n}=102)\end{array}$ \\
\hline Physical health: & & \\
A: Eyes \& ears & 9 & 74.5 \\
B: Respiratory system & 18 & 58.8 \\
C: Cardiovascular system & 13 & 46.1 \\
D: Digestive system & 18 & 83.3 \\
E: Musculoskeletal system & 8 & 21.6 \\
F : Skin & 7 & 48.0 \\
G: Nervous system & 18 & 45.1 \\
H: Genitourinary system & 11 & 81.4 \\
I : Fatigability & 7 & 52.0 \\
J : Frequency of illness & 9 & 17.6 \\
K: Miscellaneous diseases & 15 & 33.3 \\
L: Habit & 6 & 67.6 \\
Psychological health: & & \\
M: Inadequacy & 12 & 81.4 \\
N: Depression & 6 & 26.5 \\
O: Anxiety & 9 & 52.0 \\
P: Sensitivity & 6 & 53.9 \\
Q: Anger & 9 & 42.2 \\
R: Tension & 9 & 55.9 \\
\hline
\end{tabular}


psychological health complaints were about the digestive system (section D 83.3\%), and inadequacy (section $M$ $81.4 \%$ ), respectively.

The most frequently reported specific health complaints were 'need glasses to see things at a distance' concerning eye and ears complaints (67.6\% of cases), 'usually eat sweets between meals,' concerning the digestive system (63.7\%); 'menstruation causes pain,' concerning the genitourinary system (59.8\%); 'always wish someone nearby to advice,' concerning indadequacy (54.9\%); 'impossible to take regular daily exercise' concerning habits (42.2\%).

\section{Classification of neurotic traits}

Applying the recommended convention of using a total CMI score $\geq 30$ or M$\mathrm{R}$ score $\mathrm{Z} 10$ as a cutoff for indicating a degree of neurotic traits or emotional difficulties (Brodman et al. 1952), 29.4\% (by using total CMI score) or $30.4 \%$ (by using M-R score) of subjects would be regarded as neurotics (Table 2).
To classify four types of neurotic traits according to Fukamachi's criteria (1959), CMI scores of section C, I, and J were calculated and plotted on $\mathrm{Y}$-axis against M-R scores on $\mathrm{X}$-axis of the Fukamachi's discriminative chart: $9.8 \%$ of the subjects were classified as provisional neurotic (type III) and 1.0 percent as neurotic (type IV) while the remainder were normal or provisionally normal (Table 2).

Relationship between demographic variables, psychological health complaints and CMI scores on physical and overall health complaints

Two-way ANOVAs using 2 age classes $(=18,>18$ years old), 2 sibling classes (1$2,>2$ siblings), 2 residence-place classes (home, apartment), and 2 classes of M-R score $(<10,210)$ as independent variables while the CMI scores (physical and overall health complaints) were used as dependent variables. Multiple comparison between means of independent variables' classes were analyzed using Tukey's

TABLE 2.

Mean CMI and percentage of neurotics screened by Brodman and Fukamachi's criteria among Japanese female students of the present study and Sakamoto's

\begin{tabular}{lcc}
\hline & $\begin{array}{c}\text { Present study } \\
(\mathrm{n}=102)\end{array}$ & $\begin{array}{c}\text { Sakamoto's study } \\
(\mathrm{n}=109)\end{array}$ \\
\hline Mean CMI: & & \\
physical health (A-L) & $15.7 \pm 11.8$ & $14.1 \pm 9.9$ \\
psychological health (M-R) & $7.3 \pm 6.1$ & $7.7 \pm 7.3$ \\
overall health (A-R) & $23.1 \pm 15.9$ & Not available \\
Brodman's criteria: & & \\
Total score 230 & 29.4 & Not available \\
M-R score 210 & 30.4 & Not available \\
Fukamachi's criteria: & & \\
Type I : Normal & 48.0 & 57.1 \\
Type II: Provisional normal & 41.2 & 26.7 \\
Type III: Provisional neurotic & 9.8 & 12.4 \\
Type IV: Neurotic & 1.0 & 3.8 \\
\hline
\end{tabular}


Multiple Range test if significant main effects were found (SAS Institute Inc., 1990; Moore and McCabe, 1993). The results revealed significant fitting model of both physical and overall health complaints $(\mathrm{F}=2.4$, and $5.4 ; 15 \mathrm{df}$, respectively). There were significant relation between age-class, and $M-R$ score on physical health complaints ( $F=6.9$, and $17.3 ; 1 \mathrm{df}$, respectively). Similarly, there was a statistically significant relation between age-class and $\mathrm{M}-\mathrm{R}$ score $(\mathrm{F}=7.9$, and $\mathrm{F}=62.1 ; \mathrm{df}=1$, respectively): subjects aged over 18 years old reported significantly more physical health complaints or overall health complaints than the younger. Subjects who had M-R score 210 reported significantly more physical health complaints or overall health complaints than the subjects who obtained lower M$\mathrm{R}$ score (Fig. 1). There was insignificant interaction among the independent variables themselves.

\section{Discussion}

There are several reasons justifying the use of Fukamachi's discriminative chart for obtaining four types of neurotic traits in this study. Firstly, JCMI has been validated among the Japanese population, both with neurotic and healthy subjects (Fukamachi, 1959). Secondly, based on factor analysis of the J-CMI, the first factor related to items used to discriminate neurosis was designated emotional instability (Mori and Togawa, 1980). Finally, it is cautiously interpreted for applying a conventional cutoff point of the original CMI which had been validated among
non-Japanese population, to the Japanese who probably differ in terms of cultural background. Several studies showed that mean CMI were different among samples from different cultures. For example, the average CMI score of 513 Thai nursing students was $36.6 \pm 18.6$ (Nanakorn et al. 1997). Kalimo and Bice (1970), and Lin et al. (1979) have cautioned against using norms developed for data from other culture. This point can be seen from the present study, a high percentage of neurotics; $29.4 \%$, and $30.4 \%$ (using total CMI score and M-R score, respectively) classified by Brodman comparing to only $10.8 \%$ by Fukamachi's criteria.

The finding of relatively low percentages of provisional neurotics and neurotics when applying Fukamachi's criteria to this study is consistent with several studies of Japanese subjects. For example, in a study of 19 years old healthy young women by Nozawa et al. (1996), $9.2 \%$ were classified as provisional neurotics and neurotics; $11.1 \%$ of high school students were classified in these two categories (Nakada, 1992); in a study by Sakamoto et al. (1986) $12.4 \%$ and $3.8 \%$ of college students were classified as provisional neurotics and neurotics, respectively.

The other subjects reported more physical and overall health complaints than the younger, in accord with the clinical observation that, as individuals grow older, they develop more disabilities and complain more about their bodies, as found also by Brodman et al. (1953) and Abramson et al. (1965). The present study shows that psychological health complaints have some influence upon physical health complaints, sug- 
gesting the importance of further epidemiological investigation of the relationship between physical and mental illness (Robins, 1978). Several studies have shown such a relationship, for examples, Kotake et al. (1992) found that patients suffering from cardiac symptoms without any organic heart diseases may have at least in part psychosomatic background. Studies by Nozawa et al. (1996) suggested that a psychosomatic factor can influence the occurrence of orthostatic dysregulation in young women.

The type of screening used in the present study might help the detection of individuals who are having psychiatric symptoms but have not reached the stage of full blown disease. Early detection of this group could prevent the progression to a full psychiatric disorder (Ford, 1988). Thus, the group of neurotics found in the present study should receive help for their health problems by a counselling teacher. Moreover, their educational outcome such as academic mark score should be compared with that of subjects without neurotic trait to test whether a high CMI score or a neurotic trait affects the educational outcome. Future studies such as cohort or case-control design are suggested to search for causal relationship, as this cross-sectional study does not take into account the time sequence of event (Valanis, 1992).

Some of the health problems of the nursing students, such as lack of regular daily exercise, were problems of health behavior. These can be corrected not only by improved personal factors, (knowledge, attitude) but also by improved external factors such as provision of sports or exercise facilities by the educational institute.

The present finding, therefore, should provide grounds for future studies of strategies for health promotion and of preventive measures for psychological ill-health of nursing students.

\section{References}

Abramson JH. The Cornell Medical Index as an epidemiological tool. AJPH 1996; 45:287-298.

Abramson JH, Terespolsky L, Brook JG, and Kark SL. Cornell Medical Index as a health measure in epidemiological studies. Brit $\mathrm{J}$ Prev Med 1965; 19:103-110.

Brodman K, Erdmann AJ, Lorge I, and Wolff HG. Cornell Medical Index: an adjunct to medical interview. JAMA 1949; 140:530-534.

Brodman K, Erdmann AJ, Lorge I, and Wolff HG. The Cornell Medical Index-Health Questionnaire VI: the relation of patients' complaints to age, sex, race, and education. J Gerontology 1953; 8:339-342.

Brodman K, Erdmann AJ, Lorge I, Gershenson $\mathrm{CP}$, and Wolff HG. The Cornell Medical Index-Health Questionnaire III: the evaluation of emotional disturbances. $\mathrm{J}$ Clin Psychol 1952; 8:119-124.

Cronbach LJ. Coefficient alpha and the internal structure of tests. Psychometrika 1951; 16: 297-334.

Ford DE. Principles of screening applied to psychiatric disorders. Gen Hosp Psychiatry 1988; 10:177-188.

Fukamachi K. The study on the Cornell Medical Index (II): a discriminative chart as a screening test of neurotics by CMI. Fukuoka Igaku Zashi 1959; 50:3001-3009. (in Japanese)

Kalimo E, and Bice TW. Cross-cultural analysis of selected emotional questions from the Cronell Medical Index. Br J Prev Soc Med 1970; 24:229-240.

Kanehisa T, and Fukamachi K. The Cornell Medical Index. Sankyobo Co. Ltd, Kyoto, 
1988. (in Japanese)

Kotake H, Fukuki M, Miyakoda H, Hisatome I, Fujimoto $\mathrm{Y}$ et al. Cardiac neurosis: interactions between cardiac arrhythmias and symptoms using ambulatory ECG monitoring. J Medicine 1992; 23:303-313.

Lin KM, Tazuma L, and Masuda M. Adaptational problems of Vietnamese refugees. Arch Gen Psychiatry 1979; 36:955-961.

Mori T, and Togawa K. Factor analysis of Cornell Medical Index-Health Questionnaires and personality test questionnaires. Jpn J Hyg 1980; 35:486-492. (in Japanese)

Moore DS, and McCabe GP. Introduction to the practice of statistics. 2 edn, WH Freeman and Company, New York, pp 742, 1993.

Nakoda Y. A study of psychosocial factors in the psychosomatic symptoms of adolescents in Okinawa. Acta Paediatr Jpn 1992; 34:301309.

Nanakorn S, Chin-on S, Osaka R, Inutsuka H, and Fukuda K. Cornell Medical Index score observed among northeast Thai nursing students. Kurume Med J 1997; 44:99-104.

Nozawa I, Hisamatsu K, Imamura S, Fujimori I, Nakayama $\mathrm{H}$ et al. Psychosomatic aspects of healthy young women with orthostatic dysregulation. Clin Otolaryngol 1996; 21:222225.

Sakamoto K, Takao F, Yoshimoto S, Katsuno S, and Matsuura T. An epidemiologic study on the correlation between salt threshold, academic test marks, biochemical data, number of complaints, and personality in women college students. Am J Prev Med 1986; 2:351-358.

SAS Institute Inc. SAS/STAT user's guide. Version 6, 4 edn, Cary, NC, USA, Vol 2, pp 898, 944-948, 1990.

Robins LN. Psychiatric epidemiology. Arch Gen Psychiatry 1978; 35:697-702.

Valanis B. Epidemiology in nursing and health care. 2 edn, Appleton \& Lange, USA, pp 43, 1992. 